\title{
Fur Seals Display a Strong Drive for Bilateral Slow-Wave Sleep While on Land
}

\author{
Oleg I. Lyamin, ${ }^{1,2}$ Peter O. Kosenko, ${ }^{2}$ Jennifer L. Lapierre, ${ }^{1}$ Lev M. Mukhametov, ${ }^{2}$ and Jerome M. Siegel ${ }^{1}$ \\ ${ }^{1}$ Department of Psychiatry, School of Medicine, University of California Los Angeles and Neurobiology Research (151A3), Veterans Affairs Greater Los \\ Angeles Healthcare System, North Hills, California 91343 and 2Utrish Dolphinarium Ltd., Moscow, Russia 119071
}

Fur seals (pinnipeds of the family Otariidae) display two fundamentally different patterns of sleep: bilaterally symmetrical slow-wave sleep (BSWS) as seen in terrestrial mammals and slow-wave sleep (SWS) with a striking interhemispheric EEG asymmetry (asymmetrical SWS or ASWS) as observed in cetaceans. We examined the effect of preventing fur seals from sleeping in BSWS on their pattern of sleep. Four northern fur seals (Callorhinus ursinus) kept on land were sleep deprived (SD) of BSWS for 3 consecutive days, followed by 1 recovery day. EEG asymmetry was evaluated both visually and by EEG spectral analysis. SD significantly reduced the percentage of high-voltage BSWS (on average to 14\% of baseline) and REM sleep (to $60 \%$ of baseline) whereas the percentage of low-voltage BSWS was not affected. During the SD period, all seals repeatedly tried to enter BSWS (109-411 attempts per day). SD significantly increased the amount of ASWS in each seal when scored visually (to 116-235\% of baseline) and the difference in the EEG slow-wave activity (spectral power in the range of $1.2-4.0 \mathrm{~Hz}$ ) between the two hemispheres (117-197\%) as measured by the asymmetry index. High-voltage BSWS and the amount of SWS in each hemisphere were significantly elevated during the first $4 \mathrm{~h}$ of recovery. These data indicate that fur seals display a homeostatic response to the loss of SWS and that alternating SWS in the two hemispheres does not adequately compensate for the absence of BSWS.

Key words: EEG asymmetry; bilateral slow-wave sleep; slow-wave activity; sleep deprivation; sleep-wake cycle; Callorhinus ursinus

\section{Introduction}

Sleep in fur seals (pinnipeds of the family Otariidae) is unique in several aspects. Fur seals display two fundamentally different patterns of sleep. The first is bilaterally symmetrical slow-wave sleep (BSWS) and rapid eye movement (REM) sleep (Mukhametov et al., 1985; Lyamin et al., 2008a). This is the predominant pattern when fur seals sleep on land and it is similar to that seen in terrestrial mammals. The second type is characterized by a striking interhemispheric EEG asymmetry during slow-wave sleep (SWS) and a very low amount of REM sleep. This pattern is recorded when fur seals sleep in water (Lyamin and Mukhametov, 1998) and is similar to the sleep of cetaceans, which consists of unihemispheric SWS (Mukhametov et al., 1977; Mukhametov, 1984, 1987; Lyamin et al., 2004, 2008b). However, some fur seals may also display a substantial amount of SWS with interhemispheric EEG asymmetry while on land, whereas other seals are capable of displaying episodes of high-voltage BSWS (HVBSWS) while sleeping in water. It has been observed that EEG asymmetry during SWS in the fur seal is associated with (1) motion while sleeping in water and paddling with one foreflipper

Received May 4, 2008; revised July 19, 2008; accepted Sept. 13, 2008.

This work was supported by the National Science Foundation (Grant 0234687), the Defense Advanced Research Projects Agency (Grant BAA0412F8034), the Medical Research Service of the Department of Veterans Affairs, and the Utrish Dolphinarium, Ltd. We thank E. Belyaev, A. Kibalnikov, E. Nazarenko, J. Pryaslova, and 0. Shpak for their assistance during the experiments.

Correspondence should be addressed to Dr. Oleg Lyamin, Neurobiology Research (151A3), Veterans Affairs Greater Los Angeles Healthcare System, 16111 Plummer Street, North Hills, CA 91343. E-mail: olyamin@ucla.edu. DOI:10.1523/JNEUROSCI.2306-08.2008

Copyright $\odot 2008$ Society for Neuroscience $\quad$ 0270-6474/08/2812614-08\$15.00/0 and (2) brief opening of one eye which occurs during sleep both on land and in water. Flipper movements and eye opening occur contralateral to the hemisphere with low-voltage activity (Lyamin and Mukhametov, 1998; Lyamin et al., 2004).

Sleep deprivation is commonly used to study the mechanisms and functions of sleep. Total and selective sleep deprivation are two methods which lead to the loss of all stages of sleep or selective stages only (primarily REM sleep or SWS). Studies of the effects of sleep deprivation have been extensively reviewed (e.g., Rechtschaffen and Bergmann, 2002; Bonnet, 2005; Dinges et al., 2005). One of the main findings of these studies is that SWS is homeostatically regulated in mammals, in humans and likely in birds with the intensity of slow-wave activity (SWA) reflecting the duration of previous waking (e.g., Borbely and Achermann, 2005; Tobler, 2005; Newman et al., 2008; Martinez-Gonzalez et al., 2008).

The fur seal (and most likely all other otariids) can exhibit six different stages of sleep and waking: low-voltage BSWS (LVBSWS), HV-BSWS, left asymmetrical SWS (ASWS), right ASWS, bilateral REM sleep and bilateral waking. In the fur seal we can study the physiological and behavioral substrates of switching between "terrestrial" and "aquatic" types of sleep. In the current study, we examined the effects of preventing fur seals from sleeping in BSWS while on land on the occurrence of BSWS and ASWS.

\section{Materials and Methods}

Animals. Data were collected from four juvenile northern fur seals (Callorhinus ursinus; 1 female and 3 males; $20-25 \mathrm{~kg}, 2-3$ years) at the Utrish 
Marine Station of the Severtsov Institute of Ecology and Evolution (Black Sea, Russia). All procedures were approved by the University of California Los Angeles and the Veterans Affairs Greater Los Angeles Healthcare System Sepulveda Animal Research Committees, and the Commission for Bioethics of the Severtsov Institute. All studies were conducted in accordance with the National Institute of Health Guide for the Care and Use of Experimental Animals.

Surgery. Surgical procedures have been fully described in our previous publications (e.g., Lapierre et al., 2007; Lyamin et al., 2008a). Briefly, under general anesthesia (isoflurane 1-3\%) fur seals were implanted with three pairs of electrodes (stainless steel screws, $1 \mathrm{~mm}$ in diameter) for EEG recording from symmetrical fronto-occipital or fronto-parietal derivations of the right and left hemispheres. Two or three Teflon-coated multistranded stainless steel wires $(0.3 \mathrm{~mm}$ in diameter) were inserted into the neck muscles to record the electromyogram (EMG). A pair of nichrome wires $(0.5 \mathrm{~mm}$ in diameter) was placed into the orbit to record the electrooculogram (EOG). The leads were soldered to a micro-plug and attached to the skull with dental cement.

Polygraphic recording. The seals were allowed 3-6 d to recover before experiments began. On the second day after surgery all animals resumed eating fish and appeared in good condition, showing well coordinated movements and paying attention to the activity around the home pen. At this time they were placed in a pentagonal enclosure $(2 \mathrm{~m}$ per side) with a small amount of fresh sea water $(5-7 \mathrm{~cm})$ and a wooden platform $(1 \times$ $0.6 \mathrm{~m}$ ) positioned above the water in the center of the pool. Each animal was connected to a polygraph by low noise coaxial cables. EEG was recorded from two symmetrical pairs of cortical electrodes (fronto-occiptal and fronto-parietal) along with EMG and EOG continuously for $6 \mathrm{~d}$. Each seal was also continuously videotaped and observed by an experimenter located in an adjoining room. All signals were bandpass filtered (EEG and EOG at $0.3-30.0 \mathrm{~Hz}, \mathrm{EMG}$ at $0.3-70 \mathrm{~Hz}$ ), amplified, digitally sampled at $200 \mathrm{~Hz}$ and stored using CED1401 plus and Spike 2 Software (Cambridge Electronic Design, UK). During the entire period of recording, seals were fed fish twice a day (at 08:00 and 18:00) and kept on a $12 \mathrm{~h}$ light (400 lux at floor level; onset at 08:00) and $12 \mathrm{~h}$ dim light (<50 lux) cycle. During recording the average air temperature varied between 15 and $25^{\circ} \mathrm{C}$. However, for each individual seal the daily variation did not exceed $5^{\circ} \mathrm{C}$.

Sleep stages. Sleep stages were scored as in our previous studies (Lyamin et al., 2004, 2008a; Lapierre et al., 2007). EEG from symmetrical fronto-occipital derivations was scored in $20 \mathrm{~s}$ epochs for each hemisphere independently and subdivided into 3 stages: desynchronization (waking EEG), low-amplitude EEG synchronization (SWA of maximal amplitude occupies $<50 \%$ of the epoch time), or high-amplitude EEG synchronization (SWA of maximal amplitude occupies $>50 \%$ of the epoch). For each epoch an EEG stage was assigned to the left and right hemispheres. SWS was further subdivided into (1) low-voltage unihemispheric SWS (waking EEG in one hemisphere and low-voltage or highvoltage SWA in the other hemisphere) or ASWS (SWS with interhemispheric EEG asymmetry; low voltage SWA in one hemisphere and highvoltage SWA in the other hemisphere) or (2) LV-BSWS (low-voltage SWA in both hemispheres) or HV-BSWS (high-voltage SWA in both hemispheres). In this study, unihemispheric and ASWS were added together as a combined amount and will be referred to as ASWS. REM sleep was scored according to conventional criteria (bilateral desynchronization in the EEG, muscle hypotonia, occasional muscle jerks and rapid eye movements) which are generally similar in seals (Lyamin and Mukhametov, 1998; Lyamin et al., 2008a) and terrestrial mammals (e.g., Zepelin et al., 2005).

Sleep deprivation. Baseline (B), sleep deprivation (SD) and sleep recovery (SR) recordings started at 08:00. All seals were subjected to the same procedure. After a habituation period in the experimental enclosure (lasting $3 \mathrm{~d}$ ), the EEG of both hemispheres, EMG and EOG were recorded for 2 baseline days (B1 and B2). During the next $3 \mathrm{~d}$ the seals were subjected to BSWS deprivation (SD1, SD2 and SD3). Three trained observers working in shifts continuously monitored the animal. The observers aroused the seal when it displayed BSWS for a period of $10 \mathrm{~s}$ (Fig. 1). This procedure targeted HV-BSWS, whereas the amount of LV-BSWS was largely unchanged. However, the seal was not awakened if it dis- played ASWS or REM sleep. The animals were aroused by presenting an auditory stimulus: a fur seal call (one of 30 prerecorded calls was presented, each call lasting $2-5 \mathrm{~s}$, at $50-60 \mathrm{~dB}$ ). To avoid habituation, calls were presented in a randomized sequence. The seal typically displayed a clear behavioral (opening of eyes, raising of the head, changing of the posture) or polygraphic (EEG arousal in one or two hemispheres) reaction to the call. During the nighttime, the frequency of attempts to enter BSWS occasionally increased to 2-3 times per minute. Fur seal calls became less effective and the experimenters needed to enter the pool area to awaken the seal. This procedure usually prevented fur seals from entering BSWS for several minutes. After SD3, the seals were recorded for an additional $24 \mathrm{~h} \mathrm{SR}$ period during which they were not disturbed.

Data analysis. To characterize the effect of SD on the composition of SWS in fur seals, polygrams were scored visually (described in Sleep stages) and the difference between the two hemispheres in the EEG spectral power was calculated. Five second epochs were visually inspected and epochs with artifacts in the EEG were excluded from spectral power analysis. Most artifacts were associated with movements (such as walking, grooming, and postural changes). Whenever 3 or 4 of 4 consecutive $5 \mathrm{~s}$ epochs were artifact free, the spectral power in the range of $1.2-4 \mathrm{~Hz}$ (a measure of SWA) was averaged for the artifact free epochs and correlated with the visual scoring data of the corresponding $20 \mathrm{~s}$ epochs. To eliminate the effect of small differences in electrode placement on differences in EEG amplitude and power across seals and between hemispheres of each seal, SWA was standardized as a ratio to the average value in the same hemisphere during REM sleep. The expression of interhemispheric EEG asymmetry during SWS was estimated using the asymmetry index (AI) of SWA. The AI was calculated for each 20 s epoch of SWS as follows: $\mathrm{AI}=(\mathrm{L}-\mathrm{R}) /(\mathrm{L}+\mathrm{R})$, where $\mathrm{L}$ and $\mathrm{R}$ are standardized spectral power in the left and right hemispheres, respectively (spectral power in each hemisphere was divided by the average power in the same hemisphere in the same frequency range during REM sleep). To measure the degree of EEG asymmetry, the percentage of epochs with an absolute AI $>0.3$ was computed. The number of sleep epochs with an absolute AI $<0.3$ was considered an estimate of a bilaterally symmetrical EEG pattern and the number of epochs with an absolute $\mathrm{AI}>0.3$ was considered an estimate of an asymmetrical EEG pattern (for details see Lyamin et al., 2008a).

Because of technical problems several hours of recording during B1 in seals 1 and 2, respectively could not be used as baseline data. Therefore, baseline values in these seals were calculated for B2. The data collected from seals 3 and 4 during $\mathrm{B} 1$ and $\mathrm{B} 2$ are shown as an indication of the day-to-day variability of sleep parameters under baseline conditions (Table 1).

To evaluate the effect of SD on the sleep pattern, the total amount of SWS (EEG stages 2 or 3 recorded in one or two hemispheres), SWS in the left and right hemispheres, LV- and HV-BSWS and REM sleep were calculated for B2 (and will be referred as to B), SD1-3 and SR in each seal. To evaluate the effect of SD on SWA in each hemisphere, the average SWA (spectral power in the range of 1.2-4 Hz), and the percentage of SWS epochs with an absolute AI $>0.3$ were computed for the same days. To measure the effect of SD on the time course of recovery sleep immediately after the end of SD, similar calculations were performed on consecutive $4 \mathrm{~h}$ intervals (starting at 08:00) during B and SR. The duration of SWS episodes with interhemispheric EEG asymmetry (or ASWS) was also calculated for each experimental day as the number of consecutive 20 s epochs of ASWS.

Statistical analysis. All results are reported as mean \pm SEM. Sleep parameters (amount of sleep stages, SWA, duration of ASWS episodes, percentage of epochs with an absolute AI $>0.3$ ) were computed as percentage of $24 \mathrm{~h}$ or as percentage of corresponding B or SD values. Effect of SD on sleep parameters was tested by the repeated measures ANOVA (factor conditions) followed by Tukey's post hoc test. The difference between paired values (SWS parameters during B and SR) was tested by the paired $t$ test.

\section{Results}

\section{Baseline sleep parameters}

Under baseline conditions, seals spent $17-32 \%$ of $24 \mathrm{~h}$ in SWS and $2.2-7.2 \%$ of $24 \mathrm{~h}$ in REM sleep. Seals 1 and 2 spent less time 


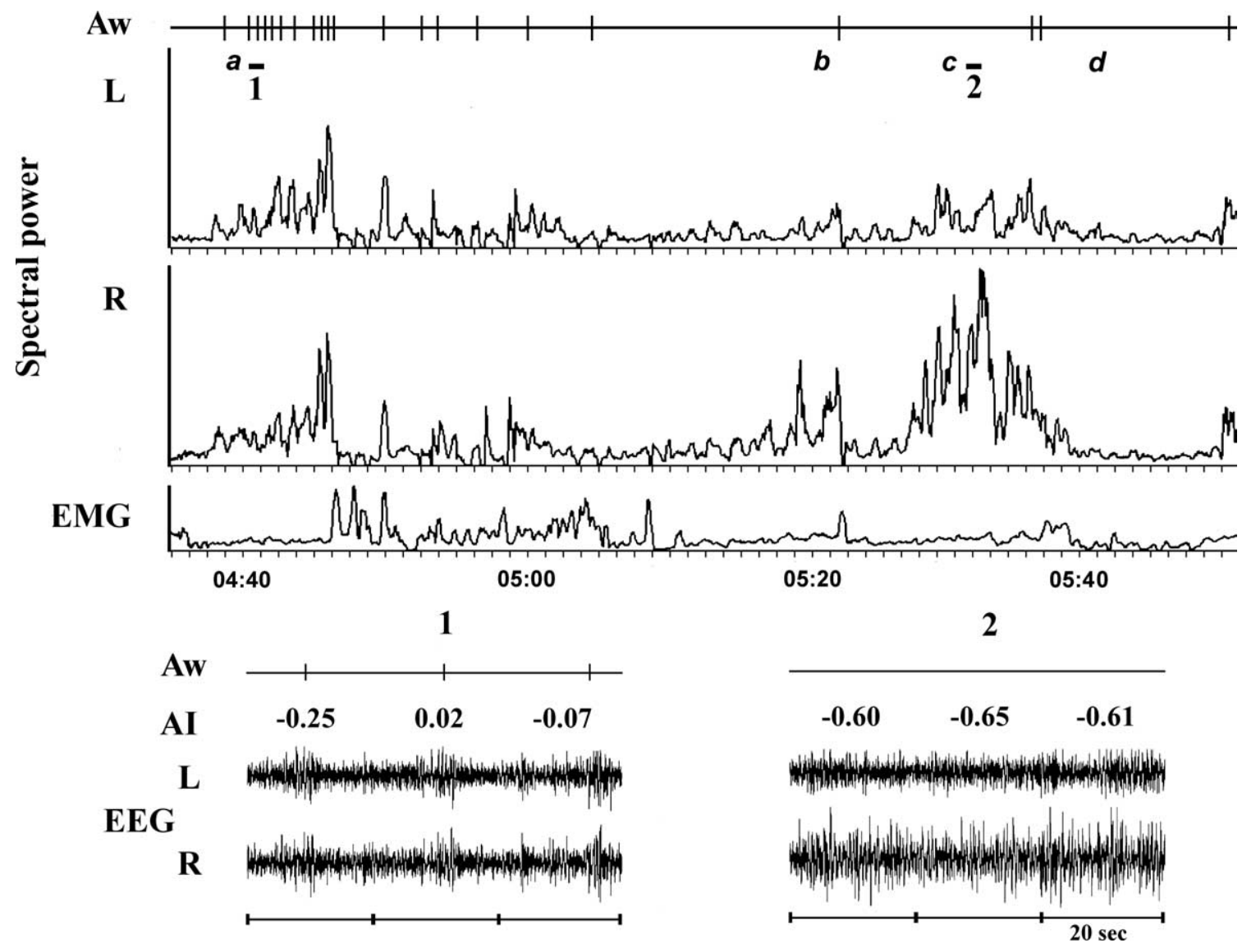

Figure 1. A representative polygraphic recording from seal 2 during deprivation of BSWS. The upper diagram shows awakenings (Aw, vertical lines), EEG spectral power in the left (L) and right (R) symmetrical fronto-occipital derivations and integrated electromyogram (EMG) values calculated for 20 s epochs. Letter $\boldsymbol{a}$ marks the beginning of a period of repeated attempts to enter BSWS. Letters $\boldsymbol{b}$ and $\boldsymbol{c}$ mark two episodes of asymmetrical SWS. Letter $\boldsymbol{d}$ marks the beginning of an episode of REM sleep. The time of recording is shown below the EMG. The lower diagram shows two $60 \mathrm{~s}$ polygrams recorded at the times marked by short lines 1 and 2 on the upper diagram and the awakenings that occurred during episode 1 . The numbers above each polygram correspond to the asymmetry index (Al) of the spectral power in two hemispheres in the range of 1.2-4 Hz in 20 s epochs (calculated as described in the methods section).

Table 1. Vigilance states and slow wave sleep parameters in fur seals under baseline conditions

\begin{tabular}{|c|c|c|c|c|c|c|c|c|c|}
\hline & \multirow{2}{*}{$\frac{\text { Seal 1 }}{\text { B2 }}$} & \multirow{2}{*}{$\frac{\text { Seal 2 }}{\text { B2 }}$} & \multicolumn{2}{|c|}{ Seal 3} & \multicolumn{2}{|l|}{ Seal 4} & \multicolumn{3}{|l|}{ Mean } \\
\hline & & & B1 & B2 & B1 & B2 & Seals 1-2 & Seals $3-4$ & Seals $1-4$ \\
\hline Waking (\% of $24 \mathrm{~h}$ ) & 78.4 & 79.6 & 75.7 & 70.9 & 63.5 & 68.5 & 79.0 & 69.6 & $74.3(2.7)$ \\
\hline SWS (\% of $24 \mathrm{~h})$ & 17.4 & 18.2 & 18.0 & 21.9 & 31.5 & 26.4 & 17.8 & 24.5 & $21.0(2.1)$ \\
\hline REM (\% of $24 \mathrm{~h}$ ) & 4.2 & 2.2 & 6.3 & 7.2 & 5.0 & 5.1 & 3.2 & 5.9 & $4.7(1.0)$ \\
\hline ASWS ( $\%$ of $24 \mathrm{~h})$ & 5.2 & 4.6 & 11.1 & 13.9 & 20.0 & 18.9 & 4.9 & 16.0 & $10.7(3.5)$ \\
\hline ASWS (\% of SWS) & 30.1 & 25.1 & 61.7 & 63.5 & 63.4 & 71.8 & 27.6 & 65.1 & $47.6(11.7)$ \\
\hline SWS epochs with AI $>0.3$ (\% of SWS) & 35.9 & 22.5 & 60.4 & 56.6 & 56.2 & 66.9 & 29.2 & 60.0 & $45.5(10.0)$ \\
\hline Episodes of ASWS $<1 \mathrm{~min}(n / \mathrm{d})$ & 56 & 58 & 62 & 82 & 100 & 72 & 57 & 79 & $67(6)$ \\
\hline $1-3 \min (n / d)$ & 15 & 9 & 21 & 34 & 36 & 26 & 12 & 29 & $21(6)$ \\
\hline$>3 \min (n / d)$ & 2 & 1 & 17 & 20 & 17 & 12 & 2 & 16 & $9(4)$ \\
\hline Maximal episode (min) & 7.7 & 7.0 & 10.0 & 10.3 & 35.7 & 19.3 & - & - & - \\
\hline
\end{tabular}

Data are presented as individual values for one (B2; seals 1 and 2 ) and two (B1 and B2; seals 3 and 4 ) baseline days and mean values (for seals 1-2, 3- 4 and all 4 seals) \pm SEM (in parentheses). Only B2 values were used to calculate mean values. SWS, Slow-wave sleep; REM, rapid eye movement sleep; ASWS, asymmetrical slow-wave sleep; Al, asymmetry index; $n / d$, number per day.

in SWS and REM sleep than seals 3 and 4 (Table 1). SWA appeared either in one hemisphere (ASWS) or in both hemispheres simultaneously (BSWS). All four seals displayed distinct episodes of ASWS (Fig. 1). During ASWS, SWA progressed in one hemisphere until it reached maximal amplitude. At the same time, the SWA in the other hemisphere was delayed and then developed until it also reached maximal amplitude. This delay varied from seconds to minutes. REM sleep usually occurred after episodes of HV-BSWS or occasionally after ASWS.

The daily amount of ASWS under baseline conditions varied considerably between seals $(4.6 \%$ of $24 \mathrm{~h}$ in seal 2 and $20.0 \%$ in seal 4), with the average for all seals being $10.7 \pm 3.5 \%$ of $24 \mathrm{~h}$ (Table 1). The AI of SWA during ASWS ranged between -0.84 and +0.87 while during BSWS it varied between -0.42 and +0.48 . As follows from the maximal AI values, some epochs scored visually as BSWS were characterized by a considerable difference in the SWA between the two hemispheres. EEG asymmetry as measured by the difference between SWA in two hemispheres (AI) also showed substantial variation between seals. The percentage of SWS epochs with an absolute AI $>0.3$ varied from 23 to $67 \%$ (Table 1). Compared with seals 1 and 2, seals 3 and 4 had a greater proportion of ASWS based both on visual scoring (a 


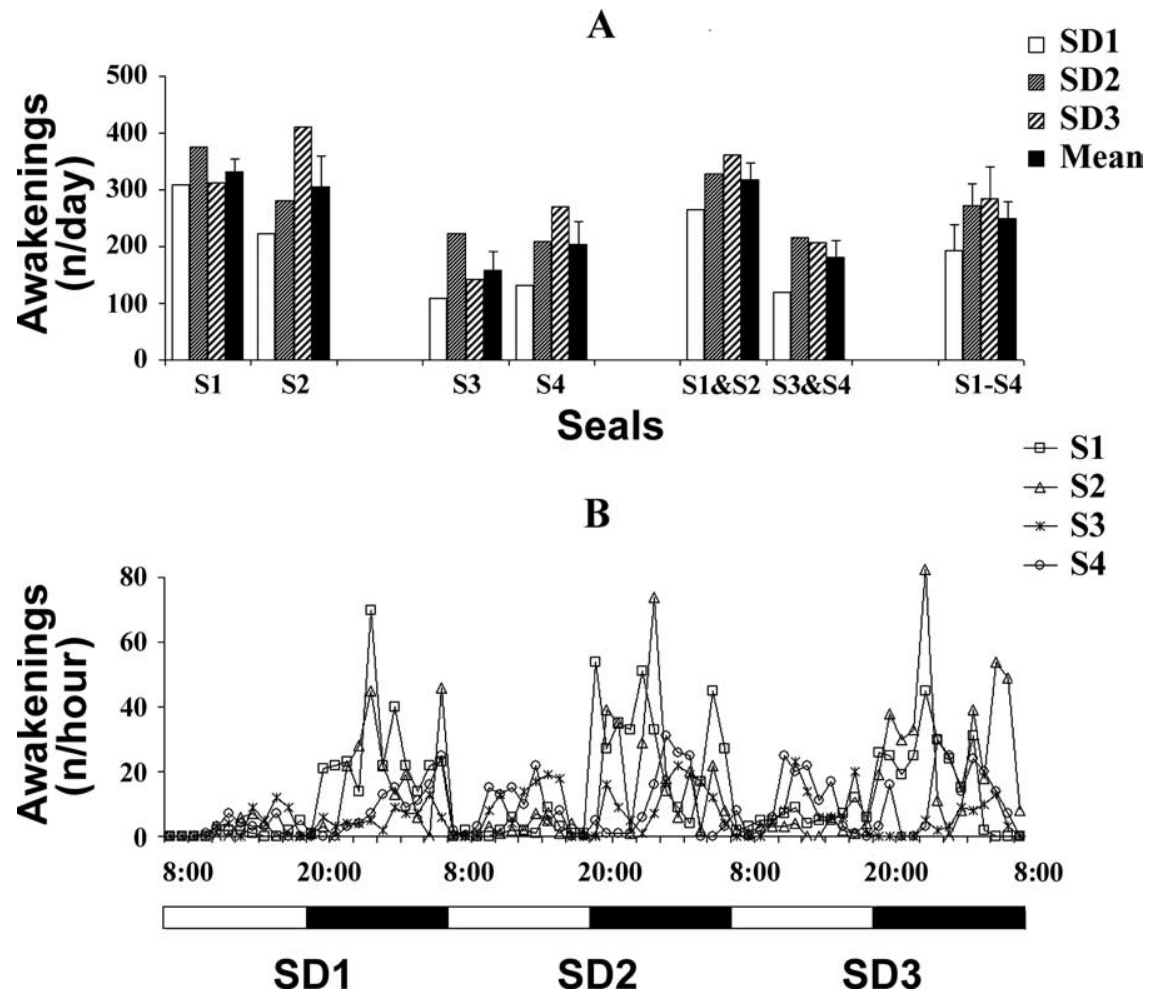

Figure 2. Number of awakenings from bilateral slow-wave sleep for each sleep deprivation day (SD1, SD2 and SD3) in fur seals (S1-S4). A, Number of awakenings per day for each sleep deprivation day (SD1, SD2 and SD3) for each fur seal (S1-S4) and the mean \pm SEM for each seal, two pairs of seals, and for all four seals combined for the entire sleep deprivation period (SD1-SD3). $\boldsymbol{B}$, Number of awakenings per hour for consecutive $1 \mathrm{~h}$ intervals during the sleep deprivation period for each fur seal (S1-S4). The horizontal bar at the bottom represents the lighting cycle (daytime is white and nighttime is black).

3.3 and 2.4 times difference, estimated as the percentage of $24 \mathrm{~h}$ and SWS, respectively) and as measured by the difference in SWA activity between the two hemispheres (a 2.5 times difference). The average proportion of SWS epochs with an absolute AI $>0.3$ was similar to the estimate of ASWS scored visually (46\% and $48 \%$ of all SWS epochs, respectively).

For all seals, the majority of individual ASWS episodes were $<1$ min (56-100 episodes per day or $60-86 \%$ of all ASWS episodes in different seals; Table 1). The number of ASWS episodes per day longer than 3 min varied greatly between seals. Seals 1 and 2 displayed only a single episode and two episodes during B, respectively, which lasted between 7 and 8 min. Seals 3 and 4 exhibited 12 and 20 episodes of ASWS per day which were longer than $3 \mathrm{~min}$. The longest episode of ASWS lasted $35 \mathrm{~min}$ and was recorded in seal 4 (Table 1 ).

In seals 3 and 4 , the difference between sleep parameters for the 2 consecutive baseline days did not exceed 25\% (Table 1).

\section{Sleep interruptions}

During the SD period seals repeatedly attempted to enter BSWS (Figs. 1,2). The number of awakenings was greater in seals 1 and 2 (with maximum values of 376 and 411 per day, respectively). These seals had the least amount of ASWS under baseline conditions. The majority of awakenings in seals 1 and 2 occurred during the nighttime (on average $90 \%$ of the daily awakenings). Seals 3 and 4 had a smaller number of sleep interruptions per day (on average $57 \%$ less than in seals 1 and 2). In this pair of seals interruptions occurred equally during both the daytime and nighttime (53 and $60 \%$ of all awakenings, respectively). All seals had the lowest number of awakenings on SD1. In two seals (2 and 4) the number of daily awakenings progressively increased throughout the SD period. In the other two seals ( 1 and 3 ) the number of sleep interruptions was maximal on SD2.

\section{Effect of BSWS deprivation on the amounts of SWS and REM sleep}

As indicated by ANOVA, SD had a marked effect on sleep in all seals (Tables 2 and 3 ). The daily amount of SWS and REM sleep were significantly affected by SD (repeated measures ANOVA, $F_{(4,12)}=9.10, p=$ 0.001 and $F_{(4,12)}=8.45, p=0.002$, respectively) (Table 2). The amount of SWS was enhanced during SR (on average 140\% of B) compared both to $\mathrm{B}$ and to all $3 \mathrm{SD}$ days. During the 3 SD days REM sleep decreased on average by $40 \%$ of B; however, the decrease was significant only for SD1. On average, there was a $30 \%$ increase in the amount of REM during SR, but this increase did not reach significance when compared with B.

The most prominent effect of SD was a decrease in HV-BSWS $\left(F_{(4,12)}=55.78, p<\right.$ 0.001 ; Table 2). During the entire SD period HV-BSWS occupied on average $<14 \%$ of the baseline level. Post hoc tests indicated significant differences between all experimental days compared both to B and SR. The SD procedure did not affect the amount of LV-BSWS $\left(F_{(4,12)}=0.56\right.$, $p=0.69)$

All SWS parameters were elevated during SR (Fig. 3). Under baseline conditions, seals were mostly active and displayed a small amount of sleep from 08:00 to 11:00. However, HV-BSWS was significantly increased during the first $4 \mathrm{~h}$ of SR (on average to 5 times the corresponding baseline value; paired $t$ test, $p<$ $0.01)$. The total amount of SWS and the amount of SWS in each hemisphere were also above baseline values $(p<0.05)$ during the first $4 \mathrm{~h}$ period of SR. SWA in each hemisphere was on average 73 and $125 \%$ greater than the baseline levels in the right and left hemispheres, respectively; however, the difference from B did not reach significance (Fig. 3). Both under baseline conditions and during SR, REM sleep was observed between 08:00 and 12:00 in only one seal. Therefore, a meaningful statistical comparison for REM sleep amounts was not possible. During the subsequent $4 \mathrm{~h}$ intervals of SR, all tested SWS parameters as well as the amount of REM sleep during the entire SR period (not shown in Fig. 3) did not differ significantly from $B$.

\section{Effect of BSWS deprivation on the composition of SWS}

For three of the four seals the amount of ASWS noticeably increased during all SD days and in the remaining seal (seal 4) it was elevated during SD2 and SD3 (Fig. 4). The increase in ASWS during SD was maximally expressed in seals 1 and 2, which displayed the smallest amount of ASWS during B ( $<4.6$ and $5.2 \%$ of $24 \mathrm{~h}$, respectively). In these seals, the amount of ASWS during the entire SD period varied between 124 and $235 \%$ of B (on average $193 \%$ ). In seals 3 and 4, which showed a greater amount of ASWS under baseline conditions (between 11 and $20 \%$ of $24 \mathrm{~h}$ ), the amount of ASWS during SD varied between 116 and 154\% of B (except for on SD1 in seal 4). On SD1 the amount of ASWS in seal 
Table 2. Vigilance states in fur seals under baseline conditions and during bilateral slow wave SD and SR

\begin{tabular}{|c|c|c|c|c|c|c|c|c|c|c|}
\hline \multirow[b]{2}{*}{ Sleep parameter } & & \multirow[b]{2}{*}{ Baseline } & \multicolumn{3}{|c|}{ Sleep deprivation } & \multirow[b]{2}{*}{ Recovery } & \multirow[b]{2}{*}{ ANOVA ( $p$ value) } & \multicolumn{3}{|c|}{ Tukey post hoc test } \\
\hline & & & Day 1 & Day 2 & Day 3 & & & SD vs B & SD vs SR & SR vs B \\
\hline \multirow[t]{2}{*}{ SWS } & $\%$ of $24 \mathrm{~h}$ & $21.0(2.0)$ & $17.8(1.8)$ & $20.7(2.5)$ & $21.9(3.1)$ & $29.1(2.8)$ & $<0.01$ & - & ${ }^{a} \mathrm{SD}_{1-3}<\mathrm{SR}^{*, * *}$ & $\mathrm{~B}<\mathrm{SR}^{*}$ \\
\hline & $\%$ of $B$ & $100(0)$ & $85.1(6.0)$ & $98.7(6.9)$ & $104.2(7.6)$ & $139.2(8.4)$ & - & - & - & - \\
\hline \multirow[t]{2}{*}{ REM } & $\%$ of $24 \mathrm{~h}$ & $4.7(1.0)$ & $2.3(0.7)$ & $3.1(1.1)$ & $3.3(1.5)$ & $6.1(1.5)$ & $<0.01$ & $\mathrm{SD}_{1}<\mathrm{B}^{*}$ & ${ }^{b} \mathrm{SD}_{1-3}<\mathrm{SR}^{*, * *}$ & - \\
\hline & $\%$ of $B$ & $100(0)$ & $46.9(8.0)$ & $64.5(11.0)$ & $68.1(19.8)$ & $132.1(7.7)$ & - & - & - & - \\
\hline \multirow[t]{2}{*}{ HV-BSWS } & $\%$ of $24 \mathrm{~h}$ & $6.1(0.6)$ & $0.8(0.4)$ & $0.6(0.4)$ & $1.1(0.5)$ & $9.2(1.3)$ & $<0.001$ & $\mathrm{SD}_{1-3}<\mathrm{B}^{* *}$ & $\mathrm{SD}_{1-3}<\mathrm{SR}^{* *}$ & $\mathrm{~B}<\mathrm{SR}^{* *}$ \\
\hline & $\%$ of $B$ & $100(0)$ & $12.1(5.8)$ & $9.4(4.4)$ & $19.1(10.0)$ & $152.5(16.8)$ & - & - & - & - \\
\hline \multirow[t]{2}{*}{ LV-BSWS } & $\%$ of $24 \mathrm{~h}$ & $4.2(1.7)$ & $3.9(1.1)$ & $3.9(0.9)$ & 4.5 (1.7) & $5.1(2.0)$ & ns & - & - & - \\
\hline & $\%$ of $B$ & $100(0)$ & $103.7(11.2)$ & $111.5(21.0)$ & 115.5 (21.4) & $117.5(23.3)$ & - & - & - & - \\
\hline \multirow[t]{3}{*}{ ASWS } & $\%$ of $24 \mathrm{~h}$ & $10.7(3.5)$ & $13.1(3.2)$ & $16.2(3.5)$ & $16.3(4.2)$ & $14.8(5.5)$ & ns & - & - & - \\
\hline & $\%$ of $B$ & $100(0)$ & $136.0(19.8)$ & $172.4(22.3)$ & $173.1(25.9)$ & $129.5(16.3)$ & ns & - & - & - \\
\hline & $\%$ of $S_{1-3}$ & $65.3(7.9)$ & $85.0(4.9)$ & $106.8(4.5)$ & $107.5(7.3)$ & $86.8(18.6)$ & $<0.05$ & - & - & - \\
\hline \multirow[t]{2}{*}{ SWS with $\mathrm{Al}>0.3$} & $\%$ of SWS & $45.5(10.0)$ & $59.5(12.4)$ & $63.4(8.5)$ & $64.0(12.3)$ & $44.1(13.3)$ & $<0.002$ & $\mathrm{SD}_{2-3}<\mathrm{B}^{* *}$ & $\mathrm{SD}_{1-3}>\mathrm{SR}^{* *}$ & - \\
\hline & $\%$ of $B$ & $100(0)$ & $132.0(4.4)$ & $150(17.0)$ & $143.2(11.8)$ & $92.0(11.0)$ & - & - & - & - \\
\hline
\end{tabular}

Data are presented as percentage of $24 \mathrm{~h}$, SWS, the second day of baseline (\% of B), or the average during 3 SD days (\% of SD $\left.{ }_{1-3}\right)$. The data are mean \pm SEM (in parentheses). SWS, Slow wave sleep; REM, rapid eye movement sleep; HV-BSWS, high-voltage bilateral SWS; LV-BSWS, low voltage bilateral SWS; ASWS, asymmetrical SWS; Al, asymmetry index; SWS with Al $>0.3$, number of SWS epochs with an absolute AI $>0.3$. ANOVA, Repeated measures ANOVA, ${ }^{*} p<0.05,{ }^{* *} p<$ 0.01. ns, not significant. ${ }^{a} \mathrm{SD}_{1}<\mathrm{SR}^{* *}, \mathrm{SD}_{2}<\mathrm{SR}^{* *}, \mathrm{SD}_{3}<\mathrm{SR}^{*} .{ }^{b} \mathrm{SD}_{1}<\mathrm{SR}^{* *}, \mathrm{SD}_{2}<\mathrm{SR}^{*}, \mathrm{SD}_{3}<\mathrm{SR}^{*}$.

Table 3. Duration of episodes of asymmetrical slow-wave sleep in fur seals under baseline conditions and during bilateral slow-wave SD and SR

\begin{tabular}{|c|c|c|c|c|c|c|c|c|c|c|}
\hline & & & Sleep dep & & & & & Tukey post & & \\
\hline Episode d & & Baseline & Day 1 & Day 2 & Day 3 & Recovery & ANOVA ( $p$ value) & SD vs $B$ & SD vs SR & R vs $B$ \\
\hline$<1 \min$ & $n / d$ & $67(6)$ & $96(16)$ & $110(24)$ & $103(13)$ & $70(20)$ & ns & - & - & - \\
\hline$<1 \min$ & $\% B$ & $100(0)$ & $146(29)$ & $159(25)$ & 155 (19) & $100(24)$ & - & - & - & - \\
\hline $1-3 \min$ & $n / d$ & $21(6)$ & $41(12)$ & $56(22)$ & $43(16)$ & $16(8)$ & $<0.02$ & $\mathrm{SD}_{2}>\mathrm{B}^{*}$ & $\mathrm{SD}_{2}>\mathrm{SR}^{*}$ & - \\
\hline $1-3 \min$ & $\% B$ & $100(0)$ & $187(12)$ & $235(56)$ & 192 (39) & 69 (19) & - & - & - & - \\
\hline$>3 \min$ & $n / d$ & $9(4)$ & $16(6)$ & $14(4)$ & $19(8)$ & $11(5)$ & ns & - & - & - \\
\hline$>3 \min$ & $\%$ B & $100(0)$ & $259(58)$ & $296(93)$ & 405 (131) & $157(55)$ & - & - & - & - \\
\hline
\end{tabular}

Data are presented as the number of episodes per day $(n / d)$ and percentage of baseline $(\% \mathrm{~B})$. The data are mean \pm SEM (in parentheses). ANOVA, Repeated measures ANOVA. ${ }^{*} p<0.05$. ns, not significant.

4 did not differ from that during B (18.8 and $18.9 \%$ of $24 \mathrm{~h}$, respectively). During SR the amount of ASWS remained elevated in 3 seals compared with B (on average $140 \%$ of B; $120-174 \%$ in the different seals). In the remaining seal (seal 2) the amount of ASWS during SR was similar to the baseline value $(4.6 \%$ of $24 \mathrm{~h}$ during $\mathrm{B}$ and $4.4 \%$ of $24 \mathrm{~h}$ during SR). ANOVA indicated that the effect of SD on the amount of ASWS was not significant $\left(F_{(4,12)}=\right.$ 2.73, $p=0.079$ ). However, the effect of SD reached significance $\left(F_{(4,12)}=3.29, p=0.048\right)$ when the amount of ASWS during B, each of 3 SD days and SR was normalized to the average amount of ASWS during the SD period (SD1-SD3). Nonetheless, the post hoc test did not show significant differences among the experimental conditions (Table 2).

Although the effects of SD on the amount of ASWS was not significant when using non-normalized data, the extent of SWA asymmetry between the two hemispheres as measured by the AI increased during SD (Fig. 5). In four seals during the entire SD period the percentage of epochs with an absolute AI $>0.3$ was between 117 and 197\% of B (on average 142\%). ANOVA indicated that the effect of SD was significant $\left(F_{(4,12)}=9.46, p=\right.$ 0.001, Table 2). Post hoc tests showed that the difference was significant between B and 2 SD days as well as between all 3 SD days and SR (Table 2). Among the studied seals, the EEG asymmetry was maximally enhanced in seals 1 and 2, which showed the smallest amount of ASWS and the smallest percentage of SWS epochs with an absolute AI $>0.3$ under baseline conditions. The smallest increase was recorded in seal 4, which had the greatest proportion of ASWS under B. During SR the percentage of epochs with an absolute AI $>0.3$ decreased in seals 1 and 2 (on average $75 \%$ of B), remained elevated in seal $3(115 \%)$ and was similar to the baseline values (103\%) in seal 4.

The amount of ASWS and the percentage of epochs with an absolute $\mathrm{AI}>0.3$ during SD were enhanced due to an increase in the duration and number of episodes of ASWS (Table 3). In all but one seal (seal 2) SD caused an increase in the frequency of short episodes of ASWS ( $<1$ min; 115-223\% compared with B). SD also induced longer episodes of ASWS. This effect was particularly evident in seals 1 and 2, which had the least amount of ASWS under B. Over the entire SD period the number of 1-3 min long ASWS episodes in these two seals increased to 2-3 times the baseline level. Moreover, under baseline conditions these seals exhibited only a single and two episodes of ASWS longer than 3 min, respectively. During SD the number of such episodes varied from 3 to 7 episodes per day (one of these episodes is shown in Fig. 1). The number of long episodes also increased in seal 4 (on average to 3 times that of $\mathrm{B}$ ). This seal showed the greatest amount of ASWS as well as the longest individual episode of ASWS under baseline conditions. During SR the number of ASWS episodes decreased. ANOVA indicated a significant effect of SD on the frequency of 1-3 min episodes of ASWS $\left(F_{(4,12)}=\right.$ 4.66, $p=0.017)$. Post hoc tests showed a significant difference between SD2 and both B and SR days in the number of these episodes (Table 3 ).

\section{Discussion}

SWS in terrestrial mammals is traditionally viewed as bihemispheric. The unusual nature of cetacean sleep (i.e., unihemispheric SWS) provides the opportunity to examine the effect of sleep loss in one cerebral hemisphere. Experiments in which dolphins were disturbed when SWA developed in a particular hemisphere and at the same time were allowed to sleep when SWA occurred in the other hemisphere, resulted in an increase of SWS during SR in the deprived hemisphere in seven of nine animals (Oleksenko et al., 1992). It was concluded that the two dolphin hemispheres are autonomous in the generation of sleep. Studies in mice, rats and in humans also showed differences in the EEG 


\section{$\square \mathbf{B}$ 『SR}

SWS

HV-BSWS
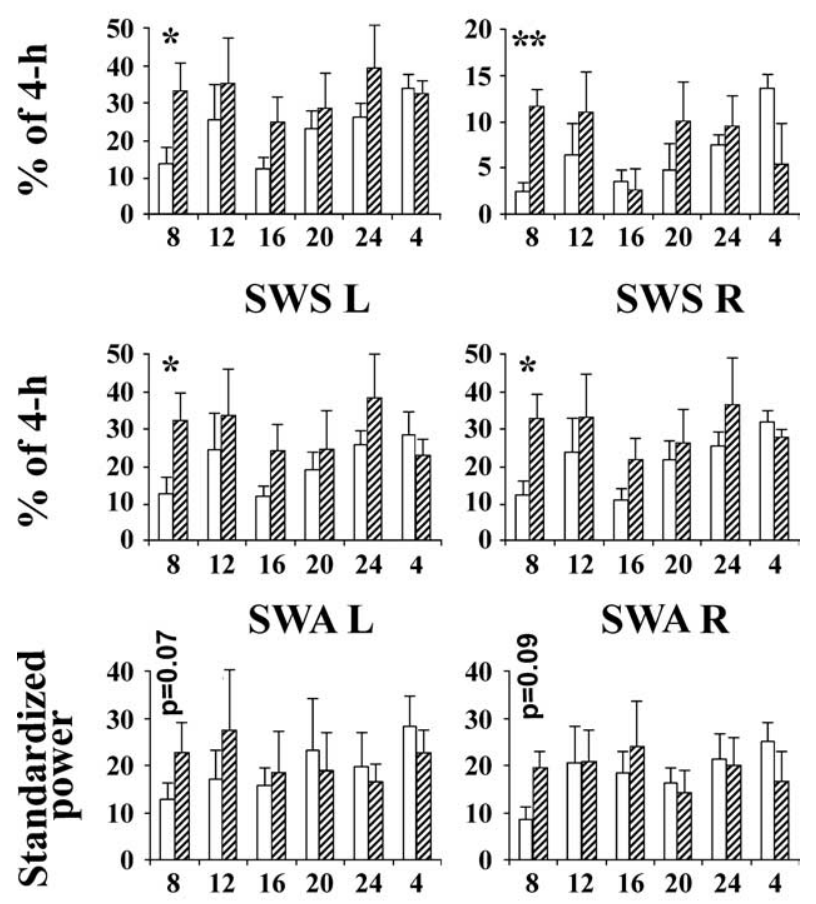

Time of day

Figure 3. Time course of sleep parameters in fur seals during baseline (B) and sleep recovery (SR). Recovery started at 08:00. The data are presented as percentage of consecutive $4 \mathrm{~h}$ intervals (the top and middle rows) or standardized spectral power in the range of $1.2-4 \mathrm{~Hz}$ (or slowwave activity, SWA). The values are shown as mean \pm SEM for all 4 seals. SWS, Slow-wave sleep. HV-BSWS, High-voltage bilateral SWS. SWS L and SWS R, SWS in the left and right hemispheres, respectively. SWA L and SWA R, SWA in the left and right hemispheres, respectively. The abscissa shows the time at the beginning hour of each $4 \mathrm{~h}$ interval. The duration of SWS, SWS $L$ and SWS R during the first $4 \mathrm{~h}$ of recovery differed significantly from baseline levels with $p<0.05\left(^{*}\right)$ whereas the duration of HV-BSWS differed with $p<0.01{ }^{* *}$ ) within the same time intervals (paired $t$ test). The difference in SWA between SR and B did not reach statistical significance in the first $4 \mathrm{~h}$ interval ( $p$ values are shown above the corresponding bars).

between symmetrical cortical areas as well as within one cortical hemisphere, indicating that EEG changes do not necessarily occur simultaneously in the entire cortex (Kattler et al., 1994; Vyazovskiy et al., 2000, 2002, 2004; Huber et al., 2004; Vyazovskiy and Tobler, 2008). These data support the idea that sleep is to some extent a local phenomenon (Krueger and Obal, 1993; Krueger et al., 1999; Tobler, 2005). The EEG asymmetry in marine mammals (cetaceans and pinnipeds) and in some birds (Rattenborg et al., 2000) is associated with asymmetrical eye state as well as with lateralized paddle activity in fur seals. This asymmetry results from a major difference in the amplitude and timing of EEG changes of the two cortical hemispheres, whereas the much smaller EEG asymmetry that has been reported in rats and humans is probably "use-dependent" in nature, being a consequence of more extensive activity of some brain tissue during the previous waking period (Kattler et al., 1994; Huber et al., 2004; Vyazovskiy and Tobler, 2008). The EEG asymmetry in terrestrial mammals and in birds is considerably less marked than that of marine mammals.

The SD procedure used in this study, being a type of selective SWS deprivation, is different from that of previous studies, in-
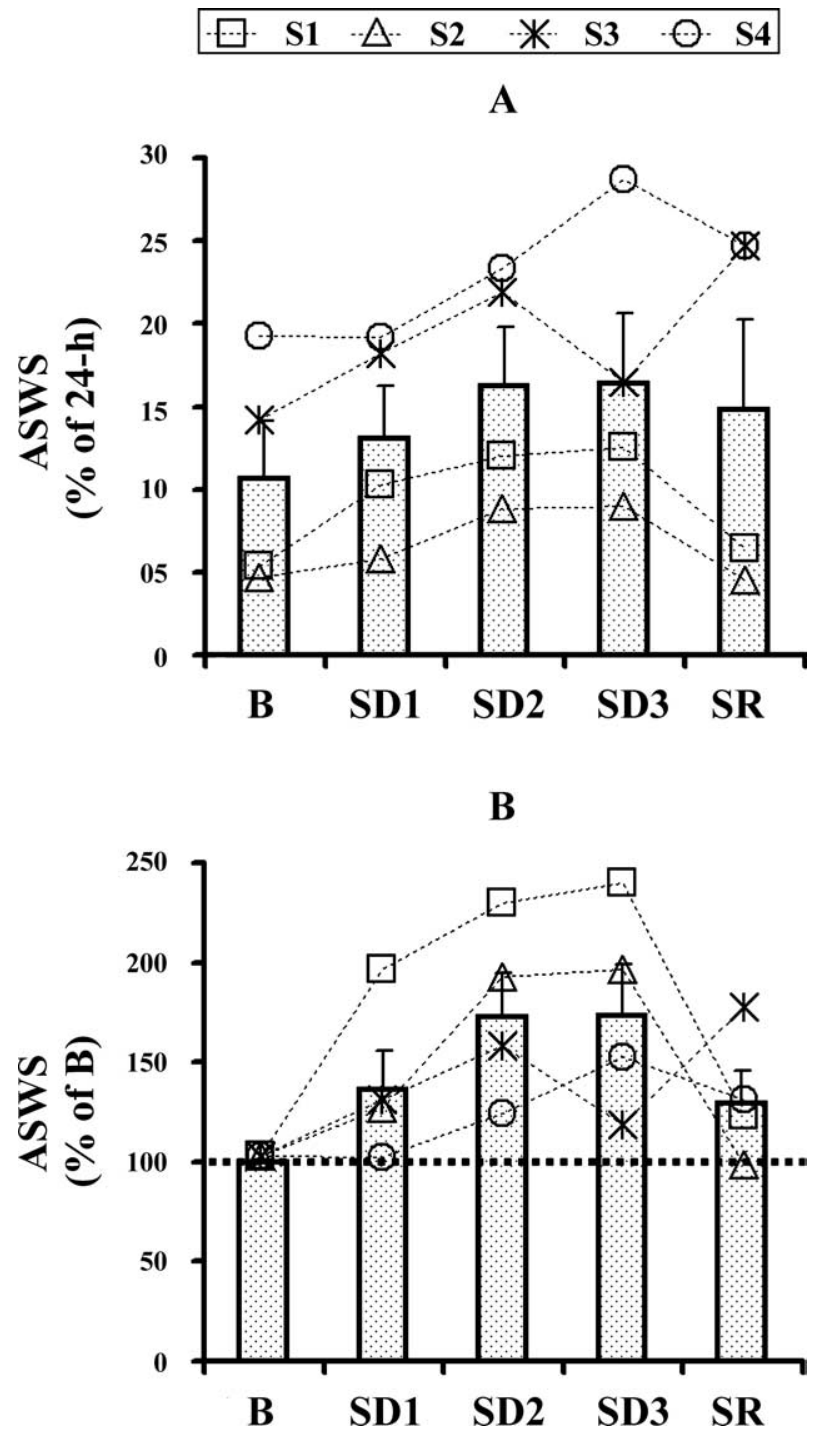

Figure 4. Amount of asymmetrical slow-wave sleep (ASWS) during baseline (B), deprivation of bilateral slow-wave sleep (SD1-SD3) and sleep recovery (SR) in fur seals (S1-S4). The values are expressed as the percentage of $24 \mathrm{~h}(\boldsymbol{A})$ or baseline $(\boldsymbol{B})$ value. The values are shown for individual seals (connected with dotted lines) and as means \pm SEM (bars).

cluding deprivation of unihemispheric sleep in dolphins. HVBSWS has never been recorded for longer than several seconds in cetaceans (Mukhametov, 1984; Lyamin et al., 2004). Pharmacologically induced HV-BSWS is incompatible with breathing in dolphins (Mukhametov et al., 1997). ASWS is the main type of sleep when fur seals stay in water. However, HV-BSWS has also been recorded in some seals in water. It is not only compatible with regular breathing but also with locomotor activity and postural control while in water. Locomotor activity and postures during BSWS are different from those during ASWS: paddling activity is less frequent and muscle tone is reduced in BSWS compared with ASWS (Lyamin and Mukhametov, 1998). During HV-BSWS both eyes are always closed whereas during ASWS one eye may briefly open, as observed in cetaceans during USWS (Lyamin et al., 2004). All these data suggest that the HV-BSWS in fur seals is a deeper state of SWS compared with ASWS. The selective SWS deprivation method used here allowed us to eliminate on average $>80 \%$ of HV-BSWS in fur seals. However, the seals were able to retain approximately the same amount (albeit fragmented) of LV-BSWS they had under baseline conditions. 


\begin{tabular}{llllllll|}
\hline & S1 & $\triangle$ & S2 & $*$ & S3 & $\odot$ & S4 \\
\hline
\end{tabular}

A

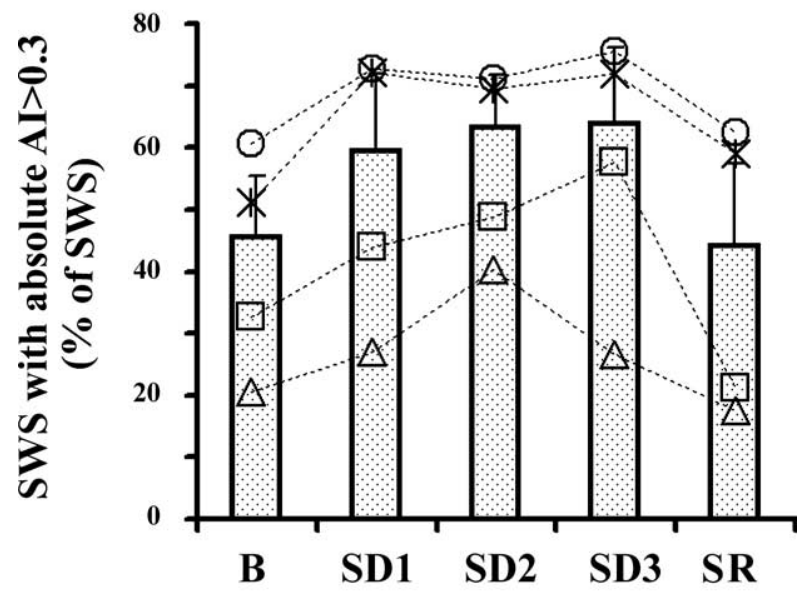

B

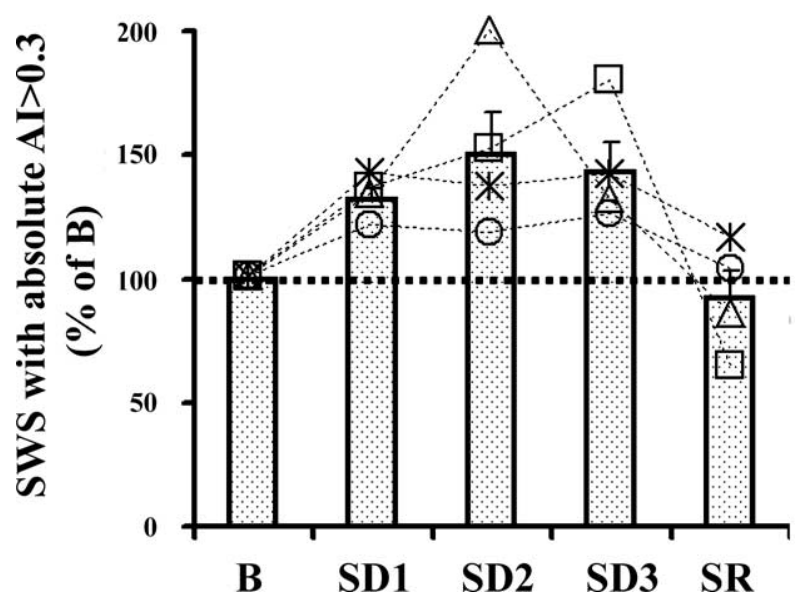

Figure 5. Percentage of slow-wave sleep epochs with an absolute asymmetry index (Al) $>$ 0.3 during baseline (B), deprivation of bilateral slow-wave sleep (SD1-SD3) and sleep recovery (SR) in fur seals (S1-S4). The values are expressed as the percentage of SWS (A) or baseline (B) value. The values are shown for individual seals (connected with dotted lines) and as means \pm SEM (bars).

There are two major findings of this study. The first is that fur seals repeatedly attempted to enter BSWS when deprived of this state while sleeping on land. This is surprising in light of our previous studies in fur seals, which showed that they can considerably reduce the amount of BSWS while remaining in water for a period of up to 2 weeks (Lyamin and Mukhametov, 1998). The second finding is that this selective SD procedure increased the amount of ASWS observed in each seal, by significantly increasing the number and the duration of ASWS episodes compared with baseline as well the extent of the EEG asymmetry. The effect of SD on EEG asymmetry was clearer when measured by the AI compared with visual scoring (Table 2). This is likely because of the limitations of the visual scoring procedure, which is based on the " $50 \%$ criteria." In many epochs scored as BSWS a difference in the amplitude of SWA between the two hemispheres could still be recognized visually.

SD not only significantly reduced the amount of HV-BSWS but also induced a compensatory response to the loss of SWS and SWA in both hemispheres during SR as shown by an increase in the amount of SWS and power of SWA in each hemisphere. This indicates that the total amount of SWS as well as and the SWS and SWA in each hemisphere are homeostatically regulated in fur seals under the conditions of our study. This is similar to what has been reported for BSWS in terrestrial mammals (for review, see Tobler, 2005) and in birds (Martinez-Gonzalez et al., 2008; Newman et al., 2008). This response was observed in all seals and was particularly evident within the first few hours after SD.

REM sleep episodes occasionally occur in fur seals after episodes of ASWS (Fig. 1) suggesting that HV-BSWS is not absolutely necessary for triggering REM sleep in this species. REM sleep was not selectively deprived in this study, however, this stage of sleep decreased during all $3 \mathrm{~d}$ of SD. Therefore, the primary cause for the decrease of REM sleep during SD appears to be the disruption of sleep and the decreased amount of HV-BSWS. It has to be also noted that REM sleep rebound was described as the most prominent response to long term total sleep deprivation in rats (Rechtschaffen and Bergmann, 2002) and in pigeons (Newman et al., 2008). Despite the decreased amount of REM sleep during SD (on average by $40 \%$ compared with B) and the increased amount of REM sleep in each seal during SR (on average by $30 \%$ compared with B), the amount of rebound of REM sleep during the $24 \mathrm{~h} \mathrm{SR}$ period in fur seals was not significantly increased, most likely because of the small sample size and variability between seals in response to SD. We can hypothesize that fur seals are capable of tolerating a greater reduction of REM sleep than rats and this would explain the very low amount of REM sleep that we recorded in seals in water for a period of 2-3 weeks (Lyamin and Mukhametov, 1998). In the wild, fur seals remain in water from 6 to 11 months per year during the pelagic (migratory) season (Gentry, 2002). The ability to tolerate a reduced amount of REM sleep appears to be characteristic of other marine mammals, including cetaceans, which do not display typical REM sleep (for review, see Lyamin et al., 2008b). Thus, in fur seals longer selective SD studies of different sleep stages would be valuable to better understand the mechanisms of sleep control in this species.

Fur seals display both BSWS and ASWS. When BSWS was interrupted, fur seals increased the frequency of ASWS episodes lasting longer than $1 \mathrm{~min}$, which were rare or absent under baseline conditions. We cannot entirely exclude the possibility that disturbance rather than sleep loss might modify the fur seal's physiological state and cause the changes in the pattern of SWS. This explanation would be consistent with our hypothesis that the proportion of ASWS in fur seals while on land is determined by the degree of the animal alertness and vigilance (Lyamin et al., 2004, 2008a). We believe it is unlikely that the procedure we used in this study causes substantial amounts of stress. Fur seals are gregarious animals (e.g., Gentry 2002). They sleep in large noisy groups on rookeries and all animals never sleep at the same time. The experimental animals were mostly awakened by calls of other seals that were similar to those that they heard before the experiments.

The baseline sleep parameters in fur seals appear to be correlated with the extent of the effect of SD on the sleep pattern. SD induced a more prominent effect in the two fur seals, which displayed less sleep overall and a minimal amount of ASWS. These seals also showed a greater pressure for HV-BSWS as evidenced by the number of attempts to enter this stage as well the amount of HV-BSWS that was not eliminated during the deprivation. The variability in the baseline sleep parameters we found in this study cannot be explained by age (all animals were $2-3$ years old) or sex (seal 1 was a female and seal 2 was a male; both displayed a smaller 
amount of SWS, REM sleep and ASWS compared with the two other male seals). Time spent in captivity and therefore the degree of adaptation to captivity also cannot explain the difference (seals 1-3 were implanted within the first month after capture whereas seal 4, which displayed the greatest amount of ASWS, spent $>1$ year in the facility before it was implanted).

To summarize, fur seals, animals which can spontaneously exhibit unihemispheric SWS, still repeatedly attempted to enter BSWS while sleeping on land. This indicates that unihemispheric SWS cannot totally substitute for bilateral SWS in fur seals under short term sleep deprivation on land.

\section{References}

Bonnet MH (2005) Acute sleep deprivation. In: Principles and practice of sleep medicine (Kryger MH, Roth T, Dement WC, eds), pp 51-66. Philadelphia: Elsevier Saunders.

Borbely AA, Achermann P (2005) Sleep homeostasis and models of sleep regulation. In: Principles and practice of sleep medicine (Kryger MH, Roth T, Dement WC, eds), pp 435-444. Philadelphia: Elsevier Saunders.

Dinges DF, Rogers NL, Baynard MD (2005) Chronic sleep deprivation. In: Principles and practice of sleep medicne (Kryger MH, Roth T, Dement WC, eds), pp 67-76. Philadelphia: Elsevier Saunders.

Gentry R (2002) The northern fur seal. In: Encyclopedia of marine mammals (Perrin WF, Wursig B, Thewissen JGM, eds), pp 813-817. San Diego: Academic.

Huber R, Ghilardi MF, Massimini M, Tononi G (2004) Local sleep and learning. Nature 430:78-81.

Kattler H, Dijk DJ, Borbély AA (1994) Effect of unilateral somatosensory stimulation prior to sleep on the sleep EEG in humans. J Sleep Res 3:159-164.

Krueger JM, Obál F (1993) A neuronal group theory of sleep function. J Sleep Res 2:63-69.

Krueger JM, Obál F Jr, Fang J (1999) Why we sleep: a theoretical view of sleep function. Sleep Med Rev 3:119-129.

Lapierre JL, Kosenko PO, Lyamin OI, Kodama T, Mukhametov LM, Siegel JM (2007) Cortical acetylcholine release is lateralized during asymmetrical slow-wave sleep in northern fur seals. J Neurosci 27:11999-12006.

Lyamin OI, Mukhametov LM (1998) Organization of sleep in the northern fur seal. In: The northern fur seal. Systematic, morphology, ecology, behavior (in Russian) (Sokolov VE, Aristov AA, Lisitzina TU, eds), pp 280 302. Moscow: Nauka.

Lyamin OI, Mukhametov LM, Siegel JM (2004) Relationship between sleep and eye state in Cetaceans and Pinnipeds. Arch Ital Biol 142:557-568.

Lyamin OI, Lapierre JL, Kosenko PO, Mukhametov LM, Siegel JM (2008a) EEG asymmetry and spectral power in the fur seal. J Sleep Res $17: 154-165$.
Lyamin OI, Manger PR, Ridgway SH, Mukhametov LM, Siegel JM (2008b) Cetacean sleep: phenotypically unusual mammalian sleep phenomenology. Neurosci Biobehav Rev 32:1451-1484.

Martinez-Gonzalez D, Lesku JA, Rattenborg NC (2008) Increased EEG spectral power density during sleep following short-term sleep deprivation in pigeons (Columba livia): evidence for avian sleep homeostasis. J Sleep Res 17:140-153.

Mukhametov LM (1984) Sleep in marine mammals. Exp Brain Res 8 (Suppl): 227-238.

Mukhametov LM (1987) Unihemispheric slow-wave sleep in the Amazonian dolphin, Inia geoffrensis. Neurosci Lett 79:128-132.

Mukhametov LM, Supin AY, Polyakova IG (1977) Interhemispheric asymmetry of the electroencephalographic sleep patterns in dolphins. Brain Res 134:581-584.

Mukhametov LM, Lyamin OI, Polyakova IG (1985) Interhemispheric asynchrony of the sleep EEG in northern fur seals. Experientia 41:1034-1035.

Mukhametov LM, Oleksenko AI, Polyakova IG (1997) The Black Sea bottlenose dolphin: the structure of sleep. In: The Black Sea bottlenose dolphin (in Russian). (Sokolov VE, Romanenko EV, eds), pp 492-512. Moscow: Nauka.

Newman SM, Paletz EM, Rattenborg NC, Obermeyer WH, Benca RM (2008) Sleep deprivation in the pigeon using the Disk-Over-Water method. Physiol Behav 93:50-58.

Oleksenko AI, Mukhametov LM, Polyakova IG, Supin AY, Kovalzon VM (1992) Unihemispheric sleep deprivation in bottlenose dolphins. J Sleep Res 1:40-44.

Rattenborg NC, Lima SL, Amlaner CJ (2000) Behavioral, neurophysiological and evolutionary perspectives on unihemispheric sleep. Neurosci Biobehav Rev 24:817-842.

Rechtschaffen A, Bergmann BM (2002) Sleep deprivation in the rat: an update of the 1989 paper. Sleep 25:18-24.

Tobler I (2005) Phylogeny of sleep regulation. In: Principles and practice of sleep medicine (Kryger MH, Roth T, Dement WC, eds) pp 77-91. Philadelphia: Elsevier Saunders,

Vyazovskiy VV, Tobler I (2008) Handedness leads to interhemispheric EEG asymmetry during sleep in the rat. J Neurophysiol 99:969-975.

Vyazovskiy V, Borbély AA, Tobler I (2000) Unilateral vibrissae stimulation during waking induces interhemispheric EEG asymmetry during subsequent sleep in the rat. J Sleep Res 9:367-371.

Vyazovskiy VV, Welker E, Fritschy JM, Tobler I (2004) Regional pattern of metabolic activation is reflected in the sleep EEG after sleep deprivation combined with unilateral whisker stimulation in mice. Eur J Neurosci 20:1363-1370.

Zepelin H, Siegel JM, Tobler I (2005) Mammalian sleep. In: Principles and practice of sleep medicine (Kryger MH, Roth T, Dement WC, eds) pp 91-100. Philadelphia: Elsevier Saunders. 